- Claudia Marquez Simões

- Emília Aparecida Valinetti

- Fernando Cardieri

Anna Maria Falcheto

Kátia Yukie

- Massako lchimura

\section{Manejo anestésico da ressecção de feocromocitoma adrenal bilateral na infância}

\author{
Instituto da Criança do Hospital das Clínicas, Faculdade de Medicina \\ da Universidade de São Paulo, São Paulo
}

INTRロDUCॄÃa

Feocromocitomas são tumores de células cromafins que captam, produzem, armazenam, metabolizam e liberam catecolaminas. A faixa etária de maior incidência é entre a terceira e quarta década de vida, no entanto, o tumor pode se desenvolver ainda na infância. A maioria dos feocromocitomas são tumores benignos tratados com remoção cirúrgica. Neste caso apresentamos a técnica anestésica empregada em um paciente submetido à ressecção de recidiva bilateral de feocromocitoma adrenal com a utilização da dexmedetomidina como medicação pré-anestésica e adjuvante anestésico endovenoso, buscando controle pressórico e estabilidade cardiovascular durante o procedimento.

RELATI DO CASD

Paciente de 8 anos, do sexo masculino, apresenta recidiva bilateral de feocromocitoma adrenal após ressecção do tumor em adrenal direita há 3 anos. $\mathrm{O}$ paciente foi readmitido no hospital com história de dor abdominal acompanhada de vômitos e crise hipertensiva. Depois de avaliação laboratorial e radiológica, que confirmaram a recidiva tumoral, o paciente foi submetido à ressecção do tumor sob anestesia geral associada à peridural contínua. Visando menor liberação de catecolaminas, associada a menor consumo de anestésicos e estabilidade cardiovascular, utilizamos a dexmedetomidina, agonista alfa-2 adrenérgico, como medicação pré-anestésica e adjuvante endovenoso durante a cirurgia. Durante a manipulação do tumor, foi necessário o uso de nitroprussiato de sódio para controle pressórico. O paciente foi extubado na sala cirúrgica, sem necessitar de drogas vasoativas, e foi encaminhado para a unidade de terapia intensiva com nebulização de oxigênio e saturação periférica de $\mathrm{O}_{2}$ de $99 \%$. Estava calmo e sem dor.

DISCUSSÃ

O manejo do paciente portador de feocromocitoma deve visar adequada manutenção da volemia e controle pressórico, se necessário, com drogas vasoativas, como alfa e betabloqueadores, e nitroprussiato de sódio. Neste caso apresentamos o emprego da dexmedetomidina, que pode ser utilizada como medicação pré-anestésica e na manutenção da anestesia, em infusão contínua para maior estabilidade cardiovascular durante a manipulação cirúrgica do tumor.

REFERÊNCIAS

. Anttila M, Penttila J, Helminen A, Vuorilehto L. Scheinin H. Bioavailability of dexmedetomidine after extravascular doses in healthy subjects. [Clinical Trial. Journal Article. Randomized Controlled Trial]. British Journal of Clinical Pharmacology. 2003;56(6):691-3.

2. Tobias JD, Berkenbosch JW. Initial experience with dexmedetomidine in paediatric-aged patients. [Journal Article]. Paediatr Anaesth. 2002;12(12):171-5.

3. Tobias JD, Berkenbosch JW, Russo P. Additional experience with dexmedetomidine in pediatric patients. Southern Medical Journal. 2003;2003,96(9):871-5.

4. Rodarte A, Diaz S, Foley J, Capparelli E. The pharmacokinetics of dexmedetomidine in post-surgical pediatric intensive care unit patients: a preliminary study. ASA Annual Meeting Abstracts. 2003;99(3A),A423.

Endereço para correspondência:

Claudia Marquez Simões

Pça Antonio Manoel do Espírito Santo, 63 - Jd. Bonfiglioli

São Paulo (SP) - CEP 05592-050

Tel. (+55 11) 9172-1271

E-mail: claucms@terra.com.br 